\title{
Crystal Structure of the Lassa virus surface glycoprotein
}

Erica Ollmann Saphire ${ }^{1,6,{ }^{*}}$ Kathryn M Hastie ${ }^{1}$, Michelle A. Zandonatti ${ }^{1}$, Luis M. Branco ${ }^{2}$, James E. Robinson ${ }^{3}$, Robert F. Garry ${ }^{4}$

${ }^{1}$ Dept. of Immunology and Microbial Science, The Scripps Research Institute, La Jolla, CA, USA

${ }^{2}$ Zalgen Labs, Germantown, Maryland, USA

${ }^{3}$ Dept. of Pediatrics, School of Medicine, Tulane University, New Orleans, LA, USA

${ }^{4}$ Dept. of Microbiology and Immunology, Tulane University, New Orleans, LA, USA

${ }^{6}$ Skaggs Institute for Chemical Biology, The Scripps Research Institute, La Jolla, CA, USA

*Correspondence to: erica@scripps.edu.

The arenavirus Lassa causes hundreds of thousands of infections in West Africa every year and severe hemorrhagic fever. The glycoprotein, GPC, is the sole antigen expressed on the viral surface and the critical target for antibody-mediated neutralization. Here we present the crystal structure of the trimeric, prefusion ectodomain of Lassa GP bound to a neutralizing antibody from a human survivor. The antibody extensively anchors two monomers together at the base of the trimer, and biochemical analysis suggests that it neutralizes by inhibiting conformational changes required for binding its intracellular receptor and for membrane fusion. This work illuminates $\mathrm{pH}$-driven conformational changes in both receptor-binding and fusion subunits of Lassa virus, illustrates the unique assembly of the arenavirus glycoprotein spike, and provides a much-needed template for vaccine design against these threats to global health. 